\title{
Modeling hologenome imbalances in inflammation and
}

\section{cancer}

\author{
Yiorgos Apidianakis ${ }^{1 *}$ and Dominique Ferrandon ${ }^{2}$ \\ ${ }^{1}$ Department of Biological Sciences, University of Cyprus, Nicosia, Cyprus \\ 2 UPR9022 CNRS, University of Strasbourg Institute for Advanced Study, Strasbourg, France \\ *Correspondence: apidiana@ucy.ac.cy
}

Edited and reviewed by:

Yousef Abu Kwaik, University of Louisville School of Medicine, USA

Keywords: Drosophila, human, mouse, innate immunity, microbiota, hologenome, diet, aging

Genetics play a pivotal role in cancer. This is best exemplified in sporadic intestinal cancer development, which usually starts with mutations in APC then in Ras, p53 and TGF $\beta$ (Sears and Garrett, 2014). Nevertheless, intestinal bacteria, diet and lifestyle contribute significantly to mucosal inflammation and cancer (Anand et al., 2008; Kostic et al., 2014; Sears and Garrett, 2014). An effective approach to study the aforementioned factors may be to analyze them combinatorially. In this regard, intestinal dysbiosis is a useful concept to describe harmful changes in the constitution of the microbiota. Another imbalance occurs during inflammatory bowel disease due to an excessive immune response to the intestinal microbiota, which in turn may lead to dysbiosis and perpetuate inflammation. Suspected factors, such as immune system mutations or tissue-damaging microbial strains, may not suffice to promote inflammation and cancer in the absence of co-founding factors that create or sustain an imbalance. Thus, a broad unifying concept may describe disease as dysfunctional interactions among environmental factors, such as diet and lifestyle, microbiota composition, and the genetic of the host. Moreover, aging affects the onset of inflammation and cancer, the host microbiota and the occurrence of sporadic mutations. Accordingly, the host genetic background and that of the microbiome, define the intestinal hologenome, which is influenced by age and the environment toward homeostasis or disease. Thus, intestinal disease may ensue when the intestinal hologenome is imbalanced, that is, when a genetically predisposed or old host interacts with its dysbiotic microbiota in an inadequate or harmful dietary or lifestyle-shaped environment.

The review and opinion articles accompanying this editorial describe key aspects of modeling the hologenome with an emphasis on intestinal infection, inflammation and cancer. One major issue discussed is the adaptation of Koch's postulates in order to assess causation between the human opportunistic pathogen Pseudomonas aeruginosa and intestinal disease in patients with cancer (Markou and Apidianakis, 2014). While Enterobacteriaceae are suspected contributors to intestinal inflammation and cancer, $P$. aeruginosa exemplifies the opportunistic nature of many bacterial species toward colonization and disease. The suggested guidelines therefore provide a simple framework within which clinical associations, experimental data, and improved outcomes upon treatment against suspected bacteria need to be taken into account in order to prove causation.
Experimental data can be obtained with the various mouse models of intestinal inflammation and cancer described comprehensively by Gkouskou et al. (2014). This review article describes the contributing role of microbiota as a whole, as well as that of specific bacterial species in exacerbating the disease. Interestingly, Enterobacteriaceae and Bacteroides species contribute to disease progression in various mouse models. In addition, intestinal dysbiosis is influenced by diet, antibiotics, and an immune genetic background conducive to exacerbated adaptive and diminished innate immune response. The authors highlight the potential of targeting the dysbiosis-inflammation-tumorigenesis axis for the development of novel therapeutic strategies for IBD and colorectal cancer.

Whereas studies on bacteria dominate the literature on the role of dysbiosis in inflammation and cancer, viruses were historically the first microbes to be linked to cancer. A modern approach to this issue is described by Iacovides and colleagues who suggest that the interplay between cancer and cell stemness can be influenced by oncogenic viruses (Iacovides et al., 2013). These viruses interfere with signaling pathways that are traditionally associated with self-renewal and lineage-commitment. Thus virus-associated cancers can serve as models to understand the link between viral infection, cancer, and stemness.

Innate immune and stress responses lie at the intersection of apoptosis and cell proliferation during inflammation and cancer. In this regard the simple model organism Caenorhabditis elegans has provided valuable insights into the tight regulation of apoptosis during development, infection, and DNA damage (Arvanitis et al., 2013). These findings have been taken a few steps further with the use of Drosophila models of infection and cancer, as reviewed by Bangi (2013). This review illustrates the key role of stress, innate immunity, and inflammatory signaling pathways in promoting intestinal stem cell proliferation and tumorigenesis. Prominent among these pathways is the c-Jun-N-terminal kinase (JNK) cascade, which in an oncogenic background can be diverted from tissue damage- or infection-mediated apoptosis to tumor cell proliferation and invasion (Apidianakis et al., 2009; Cordero et al., 2010; Bangi et al., 2012). Ligoxygakis and colleagues contribute a thorough review on Drosophila hemocytes, describing the multifaceted roles of these innate immunity cells in development, immunosurveillance, and tumorigenesis (Wang et al., 2014). Kim and Lee explain the multiple roles of Drosophila 
Duox, an NADPH oxidase, the homologs of which mediate bacterial killing via oxygen radicals in mammalian mucosae and phagocytes (Kim and Lee, 2014). The authors provide insights into the role of Duox in gut immunity, homeostasis of the intestinal epithelium, and stem cell proliferation. Complementarily, Ayyaz and Jasper put in perspective aging and three responses of Drosophila to intestinal microbes, namely, Duox, the Immune deficiency NF- $\mathrm{B}$ pathway, and the renewal of intestinal enterocytes (Ayyaz and Jasper, 2013). These two reviews provide a comprehensive analysis of intestinal dysbiosis and accompanying intestinal cell renewal, which is a homeostatic arm of the intestinal host defense induced either by pathogenic or seemingly innocuous bacteria, and showcase the usefulness of Drosophila as a model for the study of intestinal immunity, inflammation, and disease.

Regenerative and tumor-promoting cytokines in Drosophila and mammals may not necessarily emanate from tissue infiltrating blood cells (Panayidou and Apidianakis, 2013; Gkouskou et al., 2014). The review by Kux and Pitsouli highlights that regeneration signals are not confined to the Drosophila intestinal epithelium (Kux and Pitsouli, 2014). Neighboring tissues, such as muscles, trachea and potentially the neural system communicate with intestinal epithelial cells, and thus might contribute to the intestinal stem cell niche. Accordingly, regenerative or tumorpromoting inflammatory signaling may be controlled not only by tumors and their microenvironment, but also by remote organs. Taking a far-reaching perspective, Droujinine and Perrimon provide an educated guess on the tissues that may systemically provide inflammatory and other inter-organ signals either locally or systemically (Droujinine and Perrimon, 2013). The authors foresee the existence of a vast inter-organ communication network (ICN) of peptides, proteins, and metabolites that act in-between organs to coordinate cellular processes, either under homeostatic or stress conditions. A unique strength of the Drosophila model is that biochemical studies can be combined to in vivo genome-wide organ-specific genetic screens to identify ICN components.

\section{ACKNOWLEDGMENT}

Yiorgos Apidianakis is supported by Fondation Sante and Marie Curie CIG-303586.

\section{REFERENCES}

Anand, P., Kunnumakara, A. B., Sundaram, C., Harikumar, K. B., Tharakan, S. T., Lai, O. S., et al. (2008). Cancer is a preventable disease that requires major lifestyle changes. Pharm. Res. 25, 2097-2116. doi: 10.1007/s11095-008-9661-9

Apidianakis, Y., Pitsouli, C., Perrimon, N., and Rahme, L. (2009). Synergy between bacterial infection and genetic predisposition in intestinal dysplasia. Proc. Natl. Acad. Sci. U.S.A. 106, 20883-20888. doi: 10.1073/pnas.09117 97106

Arvanitis, M., Li, D. D., Lee, K., and Mylonakis, E. (2013). Apoptosis in C. elegans: lessons for cancer and immunity. Front. Cell. Infect. Microbiol. 3:67. doi: $10.3389 /$ fcimb. 2013.00067
Ayyaz, A., and Jasper, H. (2013). Intestinal inflammation and stem cell homeostasis in aging Drosophila melanogaster. Front. Cell. Infect. Microbiol. 3:98. doi: 10.3389/fcimb.2013.00098

Bangi, E. (2013). Drosophila at the intersection of infection, inflammation, and cancer. Front. Cell. Infect. Microbiol. 3:103. doi: 10.3389/fcimb.2013.00103

Bangi, E., Pitsouli, C., Rahme, L. G., Cagan, R., and Apidianakis, Y. (2012). Immune response to bacteria induces dissemination of Ras-activated Drosophila hindgut cells. EMBO Rep. 13, 569-576. doi: 10.1038/embor.2012.44

Cordero, J. B., Macagno, J. P., Stefanatos, R. K., Strathdee, K. E., Cagan, R. L., and Vidal, M. (2010). Oncogenic Ras diverts a host TNF tumor suppressor activity into tumor promoter. Dev. Cell 18, 999-1011. doi: 10.1016/j.devcel.2010.05.014

Droujinine, I. A., and Perrimon, N. (2013). Defining the interorgan communication network: systemic coordination of organismal cellular processes under homeostasis and localized stress. Front. Cell. Infect. Microbiol. 3:82. doi: 10.3389/fcimb.2013.00082

Gkouskou, K. K., Deligianni, C., Tsatsanis, C., and Eliopoulos, A. G. (2014). The gut microbiota in mouse models of inflammatory bowel disease. Front. Cell. Infect. Microbiol. 4:28. doi: 10.3389/fcimb.2014.00028

Iacovides, D., Michael, S., Achilleos, C., and Strati, K. (2013). Shared mechanisms in stemness and carcinogenesis: lessons from oncogenic viruses. Front. Cell. Infect. Microbiol. 3:66. doi: 10.3389/fcimb.2013.00066

Kim, S. H., and Lee, W. J. (2014). Role of DUOX in gut inflammation: lessons from Drosophila model of gut-microbiota interactions. Front. Cell. Infect. Microbiol. 3:116. doi: 10.3389/fcimb.2013.00116

Kostic, A. D., Xavier, R. J., and Gevers, D. (2014). The microbiome in inflammatory bowel disease: current status and the future ahead. Gastroenterology 146, 1489-1499. doi: 10.1053/j.gastro.2014.02.009

Kux, K., and Pitsouli, C. (2014). Tissue communication in regenerative inflammatory signaling: lessons from the fly gut. Front. Cell. Infect. Microbiol. 4:49. doi: 10.3389/fcimb.2014.00049

Markou, P., and Apidianakis, Y. (2014). Pathogenesis of intestinal Pseudomonas aeruginosa infection in patients with cancer. Front. Cell. Infect. Microbiol. 3:115. doi: 10.3389/fcimb. 2013.00115

Panayidou, S., and Apidianakis, Y. (2013). Regenerative inflammation: lessons from Drosophila intestinal epithelium in health and disease. Pathogens 2, 209-231. doi: 10.3390/pathogens2020209

Sears, C. L., and Garrett, W. S. (2014). Microbes, microbiota, and colon cancer. Cell Host Microbe 15, 317-328. doi: 10.1016/j.chom.2014.02.007

Wang, L., Kounatidis, I., and Ligoxygakis, P. (2014). Drosophila as a model to study the role of blood cells in inflammation, innate immunity and cancer. Front. Cell. Infect. Microbiol. 3:113. doi: 10.3389/fcimb.2013.00113

Conflict of Interest Statement: The authors declare that the study was conducted in the absence of any commercial or financial relationships that could be construed as a potential conflict of interest.

Received: 03 September 2014; accepted: 05 September 2014; published online: 24 September 2014.

Citation: Apidianakis Y and Ferrandon D (2014) Modeling hologenome imbalances in inflammation and cancer. Front. Cell. Infect. Microbiol. 4:134. doi: 10.3389/fcimb. 2014.00134

This article was submitted to the journal Frontiers in Cellular and Infection Microbiology.

Copyright (c) 2014 Apidianakis and Ferrandon. This is an open-access article distributed under the terms of the Creative Commons Attribution License (CC BY). The use, distribution or reproduction in other forums is permitted, provided the original author(s) or licensor are credited and that the original publication in this journal is cited, in accordance with accepted academic practice. No use, distribution or reproduction is permitted which does not comply with these terms. 